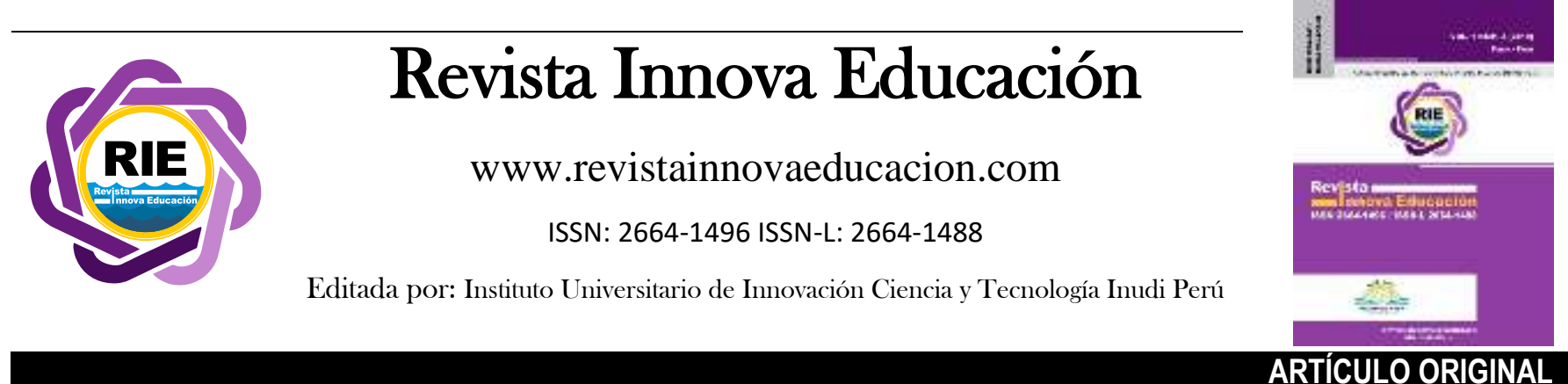

\title{
Repitencia escolar: análisis y reflexiones en un establecimiento educacional en la comuna de Valparaíso, Chile
}

\author{
School repetition: analysis and reflections in an educational establishment in the commune \\ of Valparaiso, Chile
}

Repetição escolar: análises e reflexões em um estabelecimento de ensino da comuna de Valparaíso, Chile

\author{
Ignacio Meriño-Montero ${ }^{1}$ \\ Universidad de Valparaíso, Valparaíso-Valparaíso, Chile \\ (D) https://orcid.org/0000-0002-1288-0477 \\ ignacio.merino@postgrado.uv.cl (correspondencia)
}

$\begin{array}{ll}\text { DOI (Genérico) } & : \text { https://doi.org/10.35622/j.rie.2021.01.002 } \\ \text { DOI (Documento en español) } & : \text { https://doi.org/10.35622/j.rie.2021.01.002.es } \\ \text { DOI (Document in English) } & : \text { https://doi.org/10.35622/j.rie.2021.01.002.en }\end{array}$

Recibido 15/11/2020/ Aceptado 30/12/2020 Publicado 10/01/2021

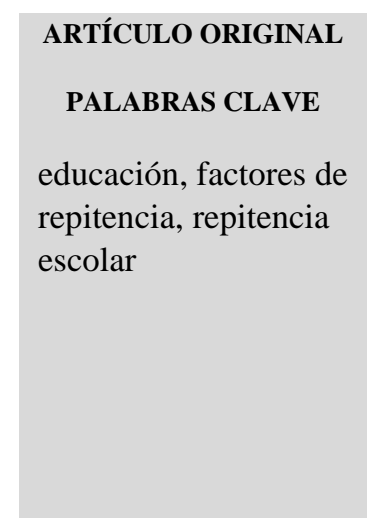

\section{KEYWORDS}

education, repetition factors, grade repetition
RESUMEN. La repitencia escolar es un fenómeno multidimensional y uno de los tres nudos críticos que presenta la educación para el cumplimiento de su universalización. Con el objeto de identificar factores de riesgo asociados en un establecimiento educacional en la comuna de Valparaíso (Chile) se realizó una investigación mixta. En ella, se analizaron y describieron fuentes primarias y secundarias de información mediante análisis de frecuencias, correlaciones, tablas de contingencia y regresión lineal sobre una base de datos de 866 estudiantes, además de análisis de contenido de entrevistas semiestructuradas a seis docentes. Los resultados establecieron que el riesgo de repitencia obedeció a factores sociodemográficos, académicos y socioemocionales, a partir de los cuales se concluyó la necesidad de trabajarlos en torno a tres componentes: apoyo familiar, acompañamiento académico y promoción de la convivencia escolar.

${ }^{1}$ Chileno, Trabajador Social y Licenciado en Trabajo Social, Universidad de Valparaíso, Chile. Magíster en Psicología Social, mención Intervención Psicosocial Comunitaria, Universidad de Valparaíso, Chile. 
which it is concluded that it is necessary to work on three components: family support, academic accompaniment, and promotion of school coexistence.

$\begin{array}{ll}\text { PALAVRAS-CHAVE } & \text { RESUMO. A repetência escolar é um fenômeno multidimensional e um dos três nós } \\ \text { críticos que a educação apresenta para o cumprimento de sua universalização. A fim } \\ \text { escolaridade, fatores } & \text { de identificar os fatores de risco associados em um estabelecimento educacional do } \\ \text { de repetência, } & \text { distrito de Valparaíso (Chile), foi realizada uma investigação mista. Nele, as fontes } \\ \text { repetência } & \text { primárias e secundárias de informação foram analisadas e descritas por meio de análise } \\ & \text { de frequência, correlações, tabelas de contingência e regressão linear em um banco de } \\ & \text { dados de } 866 \text { alunos, além da análise de conteúdo de entrevistas semiestruturadas com } \\ & \text { seis professores. Os resultados estabeleceram que o risco de repetência se deu por } \\ & \text { fatores sociodemográficos, acadêmicos e socioemocionais, dos quais se concluiu a } \\ & \text { necessidade de trabalhá-los em torno de três componentes: apoio familiar, } \\ \text { acompanhamento acadêmico e promoção da convivência escolar. }\end{array}$

\section{INTRODUCCIÓN Y ESTADO DE CUESTIÓN}

La repitencia escolar es uno de los tres nudos críticos que presenta la educación para el cumplimiento de su universalización, junto con la deserción escolar y el ingreso al sistema en edad inadecuada (Bellei, Poblete, Sepúlveda, Orellana, \& Abarca, 2013).

Si bien Chile ha experimentado un importante crecimiento sobre los años de escolaridad, los desafíos apuntan, en la actualidad, a mejorar la calidad de la educación y a disminuir las brechas existentes (Oliva, 2010). Para Donoso y Hawes (2002) la desigualdad social se expresa en desigualdades educativas frente al desarrollo de los estudiantes, a su contexto y a la sociedad en su conjunto. De esta manera, la repitencia está "vinculada al abandono y al aumento de la sobreedad (participa en la producción del fracaso escolar como un todo) provoca baja autoestima y desmotivación en los alumnos afectados, incrementa los costos del sistema educativo, y dificulta el manejo del aula" (Passolini, 2011, p. 1).

La normativa de evaluación y promoción en Chile (Decreto №522, 1997; ํ112, 1999; $\mathrm{N}^{\circ} 83$, 2001) establece que para aprobar una asignatura la nota requerida es 4,0 y la asistencia mínima de un $85 \%$. En el caso de inasistencias, las licencias médicas sólo las justifican. Para ser promovido de curso, si presentan un subsector insuficiente el promedio general debe ser igual o superior a 4,5. Si presentan dos, debe ser 5,0. En el primer ciclo básico, de primero a segundo, y de tercero a cuarto, deben ser promovidos todos los que cumplan con la asistencia ya que disponen de dos años para trabajar los contenidos. No obstante, el director del establecimiento puede, excepcionalmente, hacer repetir a un alumno si presenta un retraso significativo en lectura, escritura y/o matemática. En tercero y cuarto medio, si tiene un promedio insuficiente en Lenguaje y/o Matemática, éste debe ser igual o superior a 5,5.

Así, en el año 2018 la reprobación en enseñanza básica tuvo su punto más alto en séptimo básico con un 4,4\% y, en enseñanza media, primero medio con un 8,4\% (Chile, Ministerio de Educación [MINEDUC], 2019). Sin embargo, a partir del año 2020 un nuevo Decreto mantiene los criterios de repitencia, pero ésta pasa a ser una medida excepcional, debiendo la institución educativa fundamentar la decisión y acompañar al alumno (Decreto 67, 2018). 
Para Torres, Acevedo y Gallo (2015), la repitencia "se refiere a que algunos alumnos no aprueban los cursos respectivos y no adquieren las competencias necesarias para pasar a los niveles siguientes de formación" (p. 159), por lo que subyace la idea que el sistema podría gestionar aquellas diferencias reforzando competencias, habilidades y conocimientos que no fueron alcanzados con anterioridad (Villalobos \& Béjares, 2017). Cabe precisar que, si bien este tipo de afirmaciones abre un amplio debate sobre la efectividad de su aplicación en la política educativa, el desarrollo de la investigación no se adentrará en ella por cuanto excede a sus objetivos de realización, aun cuando sus resultados puedan contribuir a la discusión.

En consecuencia, explorar sus dimensiones en una experiencia situada posibilita comprender los factores que la inciden, dialogando con la literatura una realidad particular. De este modo, la empresa del presente artículo se aboca en identificar factores de riesgo asociados a la repitencia escolar en un establecimiento particular subvencionado de la comuna de Valparaíso, analizando y describiendo fuentes primarias y secundarias de información.

\section{Factores asociados a la repitencia escolar}

La repitencia escolar ha sido ampliamente investigada por diversos autores, coincidiendo en que sus causas no pueden ser atribuidas a un factor exclusivo (Dari, Cervini \& Quiroz, 2019; Villalobos \& Béjares, 2017; Martínez, 2016; Bellei et al., 2013; Rossano, 2006). Esto, sobre la base que en el rendimiento académico median aspectos relacionados con motivaciones y aptitudes, rol docente, vínculos interpersonales, familiares y sociales (García, Alvarado, \& Jiménez, 2000). Al respecto, el Centro UC de Estudios de Políticas y Prácticas en Educación (CEPPE, 2016), establece que el riesgo de repitencia obedece a factores sociodemográficos, académicos y socioemocionales, categorías a considerar en la comprensión de dicho fenómeno.

\section{Factores sociodemográficos}

El Tercer Estudio Regional Comparativo y Explicativo [TERCE], señala que existen “disparidades de aprendizaje asociadas al género en la mayoría de los grados, disciplinas y países evaluados (...), ventajas para las niñas en lectura y para los niños en matemática. En ciencias naturales el panorama es mixto" (Laboratorio Latinoamericano de la Evaluación de la Calidad de la Educación [LLECE] 2015, p. 80). En el ámbito local, los hombres presentan tasas de reprobación más altas que las mujeres en todos los grados de educación, siendo brechas que no superan los dos puntos porcentuales de diferencia (MINEDUC, 2019b).

Un rasgo evidente de la repitencia es el ingreso a niveles en edades inadecuadas (Bellei et al., 2013). Estudios internacionales (Schwerdt, West, \& Winters, 2015) indican que cuando se produce tempranamente disminuye las probabilidades de hacerlo a futuro. En Chile puede inferirse que estudiantes de 12 a 14 años, entre séptimo básico y primero medio, repiten más (MINEDUC, 2019). En cuanto a nacionalidad, "los estudiantes inmigrantes muestran menores resultados de aprendizaje en comparación con los niños no migrantes" (LLECE, 2015, p. 87). Lo anterior considerando que el estudiante que migra de un país a otro asumirá mayores dificultades en su adaptación al tener que asimilar el nuevo medio que lo circunda. 
Por otra parte, existe un amplio consenso en que los factores socioeconómicos influyen en las trayectorias escolares (Villalobos y Béjares, 2017; Martínez, 2016; Sánchez, Reyes y Villarroel, 2016; Puga, 2011; Dávila, Ghiardo y Medrano, 2008; Bellei, 2007). Las desigualdades apuntan a la segregación que viven las familias pues "mientras más pobres, vulnerables y excluidos son los estudiantes, mayores son sus probabilidades de no aprender lo necesario, de no alcanzar buenos desempeños, de reprobar grado, de dejar de asistir a clases, o finalmente desertar" (Román, 2013, p. 34). Los alumnos en esta situación son categorizados como Prioritarios por sus condiciones de marginación (Treviño \& Donoso, 2010). Sin embargo, dichos factores pueden ser atenuados por los colegios (Bellei, Valenzuela, Vanni \& Contreras, 2014).

Junto con lo anterior, la escolaridad de los apoderados puede explicarse, como lo señala Ikeda \& García (2014), en la medida que no participan del refuerzo escolar debido a los bajos niveles de educación formal adquiridos. Esto, a diferencia de aquellos con más estudios al convivir en ambientes más estimulantes y poseer otro tipo de interacciones apreciadas en un lenguaje diferente (Ardila, Gusmá, Matute, San Martín \& Rosselli, 2009), ya que "las condiciones estructurales y materiales de vida, las características socioeconómicas de los grupos sociales, las pautas culturales y los universos simbólicos de las familias y las comunidades educativas, determinan el desarrollo de actitudes" (Torres et al., 2015, p. 172).

\section{Factores académicos}

El ciclo vital de los estudiantes está marcado por diversos cambios y crisis (Papalia, Feldman, Martorell, Berber, \& Vásquez, 2012). Frente a esto, las transiciones de nivel las agudizan ya que "más que una continuidad o proceso natural, este tránsito se vive como un quiebre o cambio radical en la trayectoria escolar" (Román, 2013, p. 44). Según Rossano (2006), están ligados "al logro de mayores niveles de autonomía y también a otros vinculados con la organización del trabajo escolar, con los niveles de exigencia y con la inserción en un nuevo ámbito y en una nueva cultura institucional” (p. 301). Para Raczynski, Hernández, Kegevic \& Roco (2011), estos cambios generan ansiedades y preocupaciones: académicas, al poseer un mayor nivel de exigencia; procedimentales, por el manejo en el nuevo colegio; y sociales, por separarse y hacerse de nuevos amigos. Por tales motivos, "requieren de acciones y apoyos desde el colegio de acogida y de parte de la familia: estos apoyos se ven alivianados cuando el colegio de origen prepara al estudiante para la transición" (Raczynski et al., 2011, p. 19).

Alumnos repitentes presentan "más dificultades en el aprendizaje, y por ende menores niveles de logro" (Villalobos \& Béjares, 2017, p. 3), haciendo necesario contar con dispositivos pedagógicos adecuados. El Programa para la Evaluación Internacional de Estudios [PISA] 2015, indica que "cerca del 20\% de los estudiantes de los países de la OCDE no obtiene, de media, las competencias lectoras básicas" (Organización para la Cooperación y el Desarrollo Económico, 2016 , p. 4). Sumado a ello, la inasistencia a clase influye en razón que "limita e impide la exposición del estudiante al currículo, lo cual puede tener graves implicancias en su desempeño" (LLECE, 2015, p. 58). Así, la valoración y compromiso con la asistencia se asocia con un mejor rendimiento, clave en el desarrollo escolar (MINEDUC, 2018). 


\section{Factores socioemocionales}

Factores socioemocionales impactan la autoestima, motivación y aumentan la posibilidad de deserción (LLECE, 2015), tendientes a comportamintos disruptivos que pueden, inclusive, condicionar la matrícula, medida contenida en los reglamentos internos (Superintendencia de Educación Escolar, 2016). De estos surgen "relaciones tensas y desconfiadas entre unos y otros, lo que ayuda a no encontrar sentido a asistir a la escuela y colabora en la pérdida de motivación y confianza en las propias capacidades" (Román, 2013, p. 44).

En efecto, Villalobos y Béjares (2017) indican que "un niño con inestabilidad emocional suele tener problemas de concentración, afectando su rendimiento" (p. 3) ocasionando estigmatización y baja expectativa en el docente, por lo que "en tal construcción y valoración, lo que piensa, dice y hacen los docentes resulta decidor” (Román, 2013, p. 50).

Sumado a ello, los apoderados adquieren gran relevancia al significar "predisposiciones que hacen posible que un niño o una niña pueda educarse, como también a las condiciones sociales que favorecen la adquisición de dichos recursos" (Sánchez et al., 2016, p. 348). Las reuniones de apoderados, por tanto, constituyen un espacio de participación predilecto, asociadas a mejoras académicas, motivacionales y cognitivas (Valdés \& Urías, 2011). La baja participación, en cambio, las disminuye, ya que "reciben menos atención y estímulo parental respecto de sus actividades” (Espinoza, Castillo, González, \& Loyola, 2012, p. 140).

\section{Antecedentes de la experiencia}

Al aproximarnos a la comprensión de la realidad social, "el conocimiento constituye una forma de práctica social que no surge en el vacío y que debe ser analizado en un contexto que adquiere sentido desde un cúmulo de relaciones sedimentadas en un trasfondo de naturaleza semiótico-material" (Sandoval, 2013, p. 38). Sobre esta base, el desafio está en involucrarse en procesos que establezcan diálogos no excluyentes entre distintas posiciones, más que dar respuesta develada acerca de los problemas a intervenir (Montenegro, 2002).

Teniendo en perspectiva lo planteado por Montenegro (2001), el presente estudio se materializa en un establecimiento particular subvencionado en la comuna de Valparaíso, Chile, adscrito a la Ley de Subvención Escolar Preferencial y Ley de Inclusión, cuya repitencia el año 2018 fue de 7,5\%. Su proyecto educativo, de orientación humanista laica, oferta dos cursos por nivel, a excepción del quinto básico que son tres, que van desde Pre-Kínder hasta cuarto año medio Humanista - Científica. Posee una matrícula de 980 estudiantes distribuidos en 29 cursos, promediando 34 alumnos por aula. El Índice de Vulnerabilidad del Establecimiento [IVE], en relación con las características socioeconómicas de los estudiantes, es de 93,4\% en enseñanza básica y de 90,8\% en enseñanza media, con un 59,3\% de concentración de alumnos prioritarios.

Organizacionalmente, el cuerpo de trabajadores está conformado por 57 profesores, incluyendo docentes directivos y tres educadoras diferenciales, además de 31 asistentes de la educación, entre ellos, dos psicólogas y un trabajador social. De esta forma, es posible aproximarse a las posiciones de sujeto, comprendidas a partir de "articulaciones entre actores y contextos, desde donde se definen los contenidos y las orientaciones de la transformación” (Berroeta, 2011, p. 45), abordadas procedentemente. 


\section{MÉTODO}

\section{Enfoque}

Se utilizó un diseño metodológico mixto, aplicando enfoques cuantitativos y cualitativos al proporcionar evidencias más comprensivas y resultados más acabados que aquellos que se obtienen desde una sola forma de acercarse a la realidad social (Creswell \& Plano, 2010).

\section{Participantes}

Se trabajó con una base de datos de 866 estudiantes, cifra con la cual se refirió a la totalidad de la matrícula en análisis posteriores, equivalentes a los alumnos entre primero básico y cuarto medio periodo académico 2018, excluyendo la educación parvularia por responder a bases curriculares y evaluativas no pertinentes al estudio. En total fueron 25 cursos, cuyas edades fluctuaban entre los seis y 19 años, con una media de 12,1, correspondiendo un $44 \%$ de la población al género masculino y un $56 \%$ al género femenino.

Sumado a ello, fueron entrevistados seis docentes, siendo tres profesoras y tres profesores, por pertenecer al estamento encargado de transmitir los contenidos formales del currículum. Se estableció como criterio de inclusión que trabajaran en el establecimiento, desempeñarse en enseñanza básica y media, que contaran con al menos cinco años de ejercicio en la institución y que tuvieran jefatura de curso. Este último criterio, correspondió a jefaturas de octavo básico, primero y tercero medio.

\section{Técnicas de producción de datos}

Para la producción de datos se recurrió a dos procedimientos. El primero, y dado que los colegios contienen importantes volúmenes de información, la construcción de una base de datos con fuentes secundarias, utilizando referencias contenidas en libros de clases, actas de calificaciones, fichas de matrícula, entre otras. Con ellas, se elaboró un cuestionario para organizar el modo en que se ingresarían los antecedentes con el fin de "recoger información estructurada sobre una muestra de personas, utilizando el tratamiento cuantitativo y agregado de las respuestas para describir la población a la que pertenecen o contrastar estadísticamente algunas relaciones entre variables de su interés" (Meneses \& Rodríguez, 2011, p. 9).

Las variables abordadas fueron género, adoptando valor 1 para Masculino y 2 para Femenino, sumado a repitencia, condicionalidad, apoyo de equipos diferencial y psicosocial, apadrinamiento de instituciones, las que se asignaron valores 1 Si y 2 No. Respecto a las variables edad, curso, año de ingreso al establecimiento, asistencia a clases y asistencia a reuniones de apoderados se midieron como variables numéricas. Por su parte, nacionalidad se utilizó valores 1 Chilena y 2 Extranjera, dejando registro de esta última con valores 1 Boliviana, 2 Peruana, 3 Argentina, 4 Colombiana, 5 Ecuatoriana, 6 Venezolana y 7 Otra. La variable subsector con promedio insuficiente, se utilizó los siguientes valores: 1 Lenguaje, 2 Matemática, 3 Historia, 4 Ciencias, 5 Artes, 6 Inglés y 7 Otro. En el caso de caracterización socioeconómica y escolaridad del apoderado titular se operacionalizaron como variables nominales. En el caso de la primera con los valores 1 Prioritario, 2 Preferente y 3 No vulnerable, y, en la segunda, con valores 1 Sin escolarización, 2 E. Básica incompleta, 3 E. Básica completa, 4 E. Media incompleta, 5 E. Media 
completa, 6 E. Téc. Superior incompleta, 7 E. Téc. Superior completa, 8 E. Superior incompleta, 9 E. Superior completa y 10 Sin información.

El segundo procedimiento, fueron entrevistas semiestructuradas para dar cuenta de la realidad social y comprender su naturaleza (Iñiguez, 1999). Éstas responden a una conversación entre, al menos, dos partes con ciertas pautas que permiten la interacción verbal recíproca con fines profesionales (Ander-Egg, 2008). Su ventaja radicó en "adaptarse a los sujetos con enormes posibilidades para motivar al interlocutor, aclarar términos, identificar ambigüedades y reducir formalismos" (Díaz, Torruco, Martínez, \& Varela, 2013, p. 163), abordando tópicos como la percepción de causales de repitencia, rol de la familia y docente, convivencia escolar, transiciones de nivel y apoyo institucional.

\section{Análisis de datos}

Para el análisis cuantitativo, se empleó análisis de frecuencias, correlaciones y tablas de contingencia, además de regresión lineal múltiple al establecer la relación que se produce entre variables. Estos procedimientos permiten aproximarse a un modelo predictor ya que los "hechos y procesos sociales, por definición, son complejos y, en consecuencia, deben ser explicados en la medida de lo posible por la serie de variables que, directa e indirectamente, participan en su concreción" (Rodriguez \& Mora, 2001, p. 109). Los datos fueron procesados en el software SPSS versión 25.0.

En el caso de las entrevistas se realizó análisis de contenido, el cual para Andréu (2000), no se limita a la interpretación del contenido manifiesto, sino que busca profundizar su contenido latente y contexto social mediante la codificación, identificando sus características, las que son agrupadas en unidades y categorías para su descripción y análisis acabado.

\section{Consideraciones éticas}

Como refiere França-Tarragó (2005) los principios éticos, al modo kantiano, son imperativos categóricos justificados por la razón humana y se instituyen en lineamientos válidos para toda circunstancia y tiempo. Así, para asegurar el óptimo despliegue del estudio, se resguardó el nombre de los estudiantes reemplazándolo por códigos al momento de identificarlos en la estructura de variable para el diseño de la base de datos. Además, se adoptaron medidas que generaron un ambiente de confianza y respeto en el proceso de entrevistas, accediendo a ellas por intermedio de un consentimiento informado y velando por la autodeterminación de cada docente. Por último, cabe precisar que, dados los antecedentes y características del establecimiento, a juicio del autor parece irrelevante individualizarlo con su nombre ya que no menoscaba los resultados del documento, relevando así el principio de reserva.

\section{RESULTADOS}

Los resultados se dividen en dos partes. Para comenzar, se desarrolla un análisis por cada enfoque utilizado. Sobre el cuantitativo, análisis de frecuencia, correlaciones, tablas de contingencia y regresión lineal múltiple y, en lo cualitativo, análisis de contenido sobre las que se identificaron cuatro categorías: bajo involucramiento familiar, necesidad de acompañamiento en 
transiciones de ciclo, falta de expectativa docente y demanda de apoyo psicosocial. Finalmente, se presenta una discusión que integra repertorios teóricos incorporados al inicio del artículo con los resultados obtenidos en el trabajo de campo.

\section{Análisis cuantitativo}

\section{Frecuencias}

De la totalidad de los estudiantes matriculados, un 56\% correspondió al género femenino y un $44 \%$ al masculino, cuyas nacionalidades son mayoritariamente chilenas. Sólo hubo cinco estudiantes extranjeros. En cuanto a la asistencia a clases se observó que un 22,1\%, correspondiente a 191 casos, no cumplió con el mínimo determinado por la normativa de evaluación y promoción al tener una asistencia menor al $85 \%$.

En relación con los años de permanencia en el establecimiento se puede desprender que un estudiante podría alcanzar a estar un total ideal de 14 años, si se considera el desarrollo de todo su periodo escolar. Sin embargo, para la recolección de dichos datos se enfrentó la dificultad que muchas de las trayectorias no estaban en los registros internos, por lo que se estableció buscar dicha información con un margen de hasta 10 años, vale decir, hasta el periodo lectivo 2009. De este modo, 157 estudiantes se encontraban desde ese año, correspondiente a un 18,1\% y un total de 583 estudiantes los cursó, al menos, en los últimos cuatro años, representando el 43,9\% sobre el total del alumnado (ver tabla 1).

Tabla 1. Tabla de frecuencia según año de ingreso de estudiantes al establecimiento hasta el 2009

\begin{tabular}{cccc}
\hline Año de ingreso & Frecuencia & Porcentaje válido & $\begin{array}{c}\text { Porcentaje } \\
\text { acumulado }\end{array}$ \\
\hline 2009 & 157 & 18,1 & 18,1 \\
2010 & 64 & 7,4 & 25,5 \\
2011 & 62 & 7,2 & 32,7 \\
2012 & 64 & 7,4 & 40,1 \\
2013 & 76 & 8,8 & 48,8 \\
2014 & 63 & 7,3 & 56,1 \\
2015 & 97 & 11,2 & 67,3 \\
2016 & 105 & 12,1 & 79,4 \\
2017 & 63 & 7,3 & 86,7 \\
2018 & 115 & 13,3 & 100,0 \\
Total & 866 & 100,0 & 100,0 \\
\hline
\end{tabular}

Fuente: Elaboración propia en base a sistematización de datos de cuestionario

La reprobación del establecimiento fue de un 7,5\%, correspondiente a 65 estudiantes; 25 de género masculino y 40 de género femenino. Los niveles con mayores tasas de repitencia en enseñanza básica fueron séptimos años básicos lo que significó un 19,1\% dentro del nivel, y, en el caso de la enseñanza media, los primeros y terceros años medios con un porcentaje promedio de 11,1 y $11,8 \%$, respectivamente. Estos tres niveles, concentraron el $40 \%$ de los estudiantes repitentes y un $3 \%$ del total de la matrícula. 
Así mismo, 202 estudiantes tuvieron uno o más subsectores deficientes, lo que representó un 23,3\%. Al respecto, los subsectores más reprobados fueron Matemática con un 41,6\% y Lenguaje con un $37,6 \%$, sumando un porcentaje acumulado de $79,2 \%$.

\section{Correlaciones}

En consideración al desenvolvimiento de los estudiantes en términos conductuales, se recurrió a la condicionalidad como un indicador del comportamiento. A propósito, para el año 2017 hubo 29 alumnos en esa situación, de los cuales 16 correspondieron al género femenino y 13 al masculino, cifra que al año siguiente se incrementó a 41, pasando de un 3,3 a un 4,7\% de estudiantes en esta condición, aumentando considerablemente los hombres a un total de 28 . En el caso de las mujeres disminuyó a 13 estudiantes. En consecuencia, los datos arrojados mostraron que para el año 2018 la correlación entre ambas variables fue significativa, $r(866)=0,11, p=$ 0,01 .

Dado los índices de inasistencia a clases, se analizó dicha variable con la escolaridad del apoderado titular para visualizar su correspondencia. En este sentido, los datos constataron que no hubo correlación significativa entre variables $r(866)=-0,01, p=0,73$, así como tampoco fue significativa con la caracterización socioeconómica del estudiante $r(866)=0,01, p=0,71$, ni con la variable género $r(866)=-0,03, p=0,35$.

En el caso de la repitencia, si bien el género femenino representó casi dos tercios de los casos, los datos dieron cuenta que no existió una correlación al respecto ya que las variables género $\mathrm{y}$ repitencia no fueron significativas, $r(866)=-0,03, p=0,35$.

\section{Tablas de Contingencia}

Los equipos de apoyos técnicos destinados a contribuir al bienestar académico y psicosocial de los estudiantes, tales como el equipo diferencial y psicosocial, fueron variables asociadas con la repitencia. En el caso del equipo de educadoras diferenciales, pudo observarse (ver tabla 2) que focalizaron a un total de 68 estudiantes, de los cuales 11 repitieron, siendo cinco la frecuencia esperada en atención de casos. En definitiva, el 83,8\% de los estudiantes que recibieron apoyo diferencial pasó de cuso.

Tabla 2. Tabla de contingencia: Relación entre repitencia y apoyo diferencial de estudiantes matriculados en el establecimiento al 2018

\begin{tabular}{|c|c|c|c|c|c|}
\hline & & & Repitencia & No repitencia & Total \\
\hline \multirow{6}{*}{$\begin{array}{l}\text { Apoyo } \\
\text { Diferencial }\end{array}$} & Si recibe & Observado & 11 & 57 & 68 \\
\hline & & Esperado & 5,1 & 62,9 & 68,0 \\
\hline & & $\%$ de A. Diferencial & $16,2 \%$ & $83,8 \%$ & $100 \%$ \\
\hline & No recibe & Observado & 54 & 744 & 798 \\
\hline & & Esperado & 59,9 & 738,1 & 798,0 \\
\hline & & $\%$ de A. Diferencial & $6,8 \%$ & $93,2 \%$ & $100 \%$ \\
\hline \multirow{3}{*}{ Total } & & Observado & 65 & 801 & 866 \\
\hline & & Esperado & 65,0 & 801,0 & 866,0 \\
\hline & & $\%$ Total & $7,5 \%$ & $92,5 \%$ & $100 \%$ \\
\hline
\end{tabular}

Fuente: Elaboración propia en base a sistematización de datos de cuestionario 
En cuanto a la dupla psicosocial (ver tabla 3) de los 151 casos atendidos, 131 estaban distribuidos entre la enseñanza básica y enseñanza media, de los cuales quedaron repitiendo 19 estudiantes en una frecuencia esperada de 10 alumnos. La asociación entre variables indicó que el $85,5 \%$ de los estudiantes que recibieron apoyo del equipo psicosocial fueron promovidos de curso.

Tabla 3. Tabla de contingencia: Relación entre repitencia y apoyo psicosocial de estudiantes matriculados en el establecimiento al 2018

\begin{tabular}{|c|c|c|c|c|c|}
\hline & & & Repitencia & No repitencia & Total \\
\hline \multirow{6}{*}{$\begin{array}{l}\text { Apoyo } \\
\text { Psicosocial }\end{array}$} & Si recibe & Observado & 19 & 112 & 131 \\
\hline & & Esperado & 9,8 & 121,1 & 131,0 \\
\hline & & $\%$ de A. Psicosocial & $14,5 \%$ & $85,5 \%$ & $100 \%$ \\
\hline & No recibe & Observado & 46 & 689 & 735 \\
\hline & & Esperado & 55,2 & 679,8 & 735,0 \\
\hline & & $\%$ de A. Psicosocial & $6,3 \%$ & $93,7 \%$ & $100 \%$ \\
\hline \multirow{3}{*}{ Total } & & Observado & 65 & 801 & 866 \\
\hline & & Esperado & 65,0 & 801,0 & 866,0 \\
\hline & & $\%$ Total & $7,5 \%$ & $92,5 \%$ & $100,0 \%$ \\
\hline
\end{tabular}

Fuente: Elaboración propia en base a sistematización de datos de cuestionario

\section{Análisis de Regresión}

En el análisis de regresión múltiple (ver tabla 4), se observó que las variables correspondientes al año de ingreso $(\beta=-.20, t(746)=-4.55, p=.000)$, curso $(\beta=-.14, t(746)=-3.13$, $p=.002)$, condicionalidad $(\beta=.11, t(746)=-2.97, p=.003)$, caracterización socioeconómica del estudiante $(\beta=.09, t(746)=2.53, p=.012)$, asistencia a clases $(\beta=.09, t(746)=2.32, p=.021)$, apoyo diferencial $(\beta=.08, t(746)=2.18, p=.029)$ y asistencia a reuniones de apoderados $(\beta=.08, t(746)=$ $2.14, p=.033$ ) tuvieron un efecto significativo sobre la repitencia.

Tabla 4. Análisis de regresión con repitencia escolar de estudiantes matriculados en el establecimiento al 2018

\begin{tabular}{|c|c|c|c|c|}
\hline & $B$ & $E E$ & $\beta$ & \\
\hline Género &,- 01 & 0,2 &,- 042 & \\
\hline Curso &,- 01 &, 00 &,$- 14 * *$ & \\
\hline Nacionalidad & 01 & 02 & ,01 & \\
\hline Asistencia a clases & 00 & 00 &, $09 *$ & \\
\hline Año de ingreso &,- 02 & 00 &,$- 20 * * *$ & \\
\hline Condicionalidad &, 13 & 04 &, $11 * *$ & \\
\hline Apoyo diferencial & 08 & 0,4 &, $08^{*}$ & \\
\hline Apoyo psicosocial & 03 & 03 & 0,4 & \\
\hline Caracterización socioeconómica del estudiante & 03 & 01 &, $09 *$ & \\
\hline Apadrinamiento al estudiante & 01 & 03 & ,02 & \\
\hline Asistencia a reuniones de apoderado & ,01 & 01 &, $08^{*}$ & \\
\hline Escolaridad del apoderado titular & 01 & 01 &, 05 & \\
\hline$F$ total & & & & 6,18 \\
\hline$R^{2}$ Ajustado & & & & .08 \\
\hline
\end{tabular}

Fuente: Elaboración propia en base a sistematización de datos de cuestionario 
Dado lo anterior, se visualizó que llevar pocos años en el establecimiento, haber sido matriculado por primera vez en cursos más avanzados, presentar problemas de comportamiento, haber sido categorizado como estudiante prioritario y tenido baja asistencia, contar con apoyo del equipo de educadoras diferenciales por presentar necesidades educativas especiales y que el apoderado no haya asistido, o bien tuviera baja asistencia a las reuniones, aumentaría las probabilidades el repetir de curso.

\section{Análisis cualitativo}

\section{Bajo involucramiento familiar}

Docentes identificaron la relevancia que tienen los padres, madres y apoderados en el proceso formativo de sus pupilos, y la influencia que ejercen en sus itinerarios escolares. De este modo, existe un correlato entre las dinámicas familiares con el desenvolvimiento académico, horas de estudio y la estabilidad emocional, por lo que un bajo involucramiento con el establecimiento impediría desplegar todas esas potencialidades.

Tiene que ver con algún nivel, algún grado de abandono, pero no en el abandono de... la... del abandono académico de los chicos, el no seguimiento de las familias que tienen alguna implicancia es como la falta de estructura, la poca estructura que pueden tener algunas familias (Docente C).

Problemas personales siguen persistiendo, de repente continúan igual po', les afecta mal po', pero no sé si la repitencia en sí, yo creo que siguen enfocando en su tema familiar y eso no los deja avanzar, entonces quedan como estancados (Docente A).

La desesperanza de los apoderados influye en el desempeño escolar ya que existen diferencias en la proyección de capitales culturales con sus hijos, generando brechas con expectativas que desencadenan sentimientos de desánimo y presión a ellos mismos. Sin embargo, esta relación es bidireccional, en cuanto el rendimiento del estudiante permea la dinámica de su familia y lo que pasa al interior de ésta impacta en el alumno.

Las realidades que hemos comenzado a ver ahora son más complejas, por ende, muchas veces los chicos que están estudiando ahora son los primeros que van a entrar, o que pretenden entrar a una carrera universitaria... entonces familiarmente no se ve como una realidad tan tangible, y muchas veces se colocan muchas esperanzas en los chiquillos, y los chiquillos tienden a frustrarse más rápidamente (Docente $\mathrm{B}$ ).

Cuando un cabro repite hay un quiebre en su historial académico, y ese quiebre de ese historial académico no es del chiquillo, es de la familia... (Docente C).

De este modo, el acompañamiento del apoderado se expresa bajo criterios de exigencia confrontacionales, reduciendo el interés del estudiante por sus quehaceres académicos además de tensionar las relaciones que circulan en torno al colegio. Los constantes refuerzos negativos y poco asertividad en el empleo del lenguaje afectan anímicamente a los alumnos disminuyendo su percepción de autoestima.

Si hay un refuerzo negativo el chico muchas veces tiende a convencerse de que no va a ser capaz, nuevamente, y lo más probable es que vuelva a repetir (...) un rol más estricto que 
motivador, como un rol más de fiscalizar, de exigir, de ver... ¿hiciste la tarea, ¿cómo te fue en la prueba?... pero no hay una ayuda de...trajiste la tarea, ya, en qué te puedo colaborar, o ¿qué aprendiste hoy? (Docente B).

\section{Necesidad de acompañamiento en transiciones de ciclo}

Las transiciones están acompañadas de cambios en las etapas del ciclo vital, y la estructura organizacional se enfrenta a desafíos en función del cumplimiento del currículum. Esto, conlleva a una desestabilización de los estudiantes que ven interrumpida la continuidad en sus procesos formativos, y sobre el cual no se visualiza un trabajo institucionalizado para dicha adaptación como resultado de la complejidad de los contenidos, el aumento de docentes por subsector y cambios de estos a medida que avanzan de curso.

De primero a cuarto básico son los mismos, de quinto a octavo son los mismos, de primero a cuarto son los mismos, pero, por etapa, cambian todos los profes po'. Pa' máh remate, existe una brecha entre cuarto y quinto básico en donde te cambian todos los profesores. (...) el niño, además de estarse acostumbrando a poder entender ciertas cosas, además se tiene que acostumbrar al tipo del trabajo del profesor (Docente A).

En suma, se identificó marcadas diferencias en las transiciones que se dan en enseñanza básica y en enseñanza media. La primera, relacionada con el paso a la adolescencia y, consecuentemente, con los cambios físicos, psicológicos, sociales y hormonales que experimentan, percibidos como una dificultad dado los intereses que manifiestan los estudiantes. Por su parte, el inicio de la enseñanza media, demanda adaptaciones con la configuración del curso, el cual advierte cambios por la renovación del alumnado y en el afrontamiento de nuevas lógicas de trabajo que se distancian del ciclo básico anterior.

El primero medio es por todo el cambio, como la reestructuración del curso, donde tienen que aprender a conocerse, muchos se van, otros llegan con otras realidades, otros cuentos... es más como un proceso de adaptación en donde se va como conociendo ahí y adaptando (...) los más chiquititos, que serían los sextos y los séptimos, es por los cambios hacia la adolescencia, es decir, se nota mucho en donde hay algunos que aún son muy niños, otros donde ya comienzan a despertar el que le gusta a alguien, entonces son dos extremos (Docente B).

\section{Falta de expectativa docente}

El rol del docente en el desempeño escolar de los estudiantes resulta clave más allá de la mera transmisión de conocimientos. La vinculación que se establece puede proporcionar un clima adecuado o no para atender las necesidades que subyacen de sus resultados académicos, por lo que tener comprensión del contexto sociofamiliar tendría un alcance en el rendimiento del alumno.

Mucho tiene que ver con eso po', mucho tiene que ver cómo tú funcionas familiarmente, mucho tiene que ver con todo lo externo o el contexto alrededor del cabro chico, pero además todas las herramientas que tú le puedas entregar al niño (Docente F).

Las preconcepciones que tienen los docentes sobre los estudiantes trazan el tipo de relaciones. En este sentido, fue posible visualizar el reconocimiento de etiquetas a alumnos con 
bajo desempeño y en situación de repitencia, estigmatizándolos y generando condiciones que se traducen en diferenciaciones evaluativas y de mayor exigencia. Lo anterior, desde una relación que es regulada por el rendimiento escolar del estudiante.

Dicen como que... él no, es que él ya repitió, entonces a él hay que verlo, darle unas menos oportunidades, no lo hizo a la primera, entonces no (...) como que tiene que sentirse bendecido porque tuvo la oportunidad de quedarse (Docente B).

Hablar del repitente es como... antes se hablaba del sidoso, del marihuanero de, no sé, es una etiqueta que guarda al cabro y no vuelve... y es una competencia consigo mismo que muchas veces las condiciones para poder tener una vida académica normal, entre comillas, no las tuvo, no las tuvo no más (Docente C).

\section{Demanda de apoyo psicosocial}

Las demandas personales, familiares y sociales se expresan en la no satisfacción de mínimos que permitan desenvolverse adecuadamente en el espacio escolar, carenciados en diversos aspectos relacionados con el ámbito socioemocional de los alumnos, los que requieren de un acompañamiento especializado en conjunto con el quehacer docente.

La situación socioemocional del estudiante se viene prolongando desde mucho tiempo atrás, entonces en todo ese proceso... la autoestima se ve evidentemente deteriorada... y creo que los colegios y los profesores hacemos bien poco por contrarrestar eso en realidad (Docente D).

Si bien la incorporación de equipos técnicos ha presentado resistencias históricas entre los profesores al configurar un nuevo ámbito de acción en las escuelas, dicha dimensión ha ido adquiriendo, paulatinamente, mayor visibilidad, sensibilizando la acción pedagógica y proporcionando nuevas herramientas para la gestión del ejercicio docente.

Ahora se comienza como un foco hacia más darle una cierta estabilidad emocional primero a los niños, y luego lo académico. Antes era lo académico lo central y lo emocional venía como prácticamente estabilizado dentro de sus propias realidades, pero ahora los problemas que se ven son más complejos (Docente B).

\section{DISCUSIÓN}

El recorrido del presente artículo permite visualizar el fenómeno de la repitencia escolar como expresión de sus dimensiones sociodemográficas, académicas y socioemocionales, situadas en un contexto y espacio determinado en que se desarrollan (Sandoval, 2013).

En lo que respecta a la primera dimensión, y a diferencia con la tendencia a nivel nacional que muestra una mayor reprobación en los hombres (MINEDUC, 2019b), en el establecimiento son las mujeres quienes más repiten. No obstante, los resultados indican que no existen diferencias estadísticamente significativas con la repitencia, por lo que la variable género no tiene incidencia en este aspecto. 
Caso contrario ocurre con la caracterización socioeconómica, puesto que impacta en los estudios, concordando con la percepción docente y con lo expresado por diversas fuentes consultadas al señalar que, a mayor vulnerabilidad, existen mayores probabilidades de repetir de curso (Villalobos y Béjares, 2017; Martínez, 2016; Sánchez et al., 2016; LLECE, 2015; Román, 2013; Dávila et al., 2008).

Junto con lo anterior, si bien es posible señalar que la escolaridad del apoderado no determina condiciones de repitencia, esta sí tiene incidencia en el estudiante. Al respecto, una baja escolarización repercute en los capitales culturales, materiales y simbólicos de las_familias, implicando, a su vez, menores expectativas de las trayectorias escolares, así como de los apoyos personales y académicos brindados (Ardila et al. 2009). Lo anterior, propende al debilitamiento del rol de apoderado y su vinculación con las actividades del establecimiento.

En lo académico, la literatura coincide con que la baja asistencia a clases es una variable que aumenta las posibilidades de reprobar (MINEDUC, 2018; LLECE, 2015), encontrando correspondencia entre la asistencia y el rendimiento. En el caso de los apoderados, la escasa presencia en las reuniones tendría similar repercusión, puesto que configuran un eje central en la vida de los niños, niñas y jóvenes (Torres et al., 2015). Por tal motivo, trabajar en función de disminuir los porcentajes de ausentismo, tanto en apoderados a las reuniones como en estudiantes a clases, se torna relevante.

En tal sentido, y al igual como ocurre a nivel país, la repitencia se intensifica en séptimos básicos y primeros medios (MINEDUC, 2019), además de tercero medio. Sobre estos cursos, los datos arrojados están por sobre el promedio nacional. No obstante, la repitencia más que un acontecimiento dado por no alcanzar una calificación y/o asistencia, es la expresión continua de una trayectoria previa, por lo que los años anteriores resultan elementales para su comprensión y para el desarrollo de acciones posteriores.

En cuanto a las transiciones por cambios de ciclo, éstas afectan el rendimiento escolar y son profundizadas por las etapas de desarrollo en que se encuentran los estudiantes (Papalia et al., 2012). Ellas manifiestan dificultades en términos de sus intereses y las crisis por las que atraviesan, siendo un factor visualizado por los docentes, teniendo un correlato con los planteamientos teóricos (Román, 2013; Raczynski et al., 2011; Rossano, 2006). En este sentido, el comportamiento de los estudiantes también se constituye como factor en el desempeño escolar (Villalobos y Béjares, 2017) con un doble alcance. Por un lado, incide en el rol docente por cuanto estigmatiza al estudiante y disminuye las expectativas hacia ellos, tensionando la relación entre ambos y, en segundo lugar, denotan dificultades en las relaciones interpersonales con sus pares, las cuales pueden afectar su autoestima, motivaciones e inestabilidad emocional, siendo la manifestación de un problema aún mayor.

Finalmente, la demanda de apoyos técnicos por parte de los docentes hace imprescindible reconocer el fenómeno de la repitencia como producto de una confluencia de factores que llevan a ella, haciendo patente la necesidad de gestionar las desigualdades para la consecución de los objetivos académicos. Para ello, se debe contar, además, con la especificidad de los distintos dispositivos al interior del establecimiento, sumando no tan solo a profesores, sino también al resto 
de la comunidad educativa, fomentando y fortaleciendo, permanentemente, la participación de padres, madres y apoderados.

En síntesis, dado los hallazgos de la lectura teórica y la recolección y análisis de datos es posible sostener que en la repitencia; sociodemográficamente, el género no influye y que las variables que inciden están dadas a partir de una mayor vulnerabilidad socioeconómica, débil acompañamiento familiar y baja vinculación con el establecimiento; académicamente, una baja asistencia a clases y cambios por transiciones de ciclo tienen relación, y; socioemocionalmente, la falta de expectativa docente, problemas de convivencia escolar, así como la necesidad de apoyo psicosocial a los estudiantes resultan preponderantes.

\section{CONCLUSIONES}

Los resultados muestran la importancia de gestionar los hallazgos en sus dimensiones sociodemográficas, académicas y socioemocionales, incorporando la perspectiva situada para poder intervenir las tasas de repitencia del establecimiento orientada en tres componentes; apoyo familiar, acompañamiento académico, y; promoción de una buena convivencia escolar.

El primero componente, en función de fortalecer y empoderar el rol de los padres, madres y apoderados en la formación de sus pupilos. Estrechar vínculos de éstos con el establecimiento permitirá robustecer condiciones sociales que favorezcan la adquisición de diversos recursos.

El segundo, centrado en el acompañamiento académico hacia los estudiantes que propenda a un seguimiento efectivo a sus itinerarios escolares. En este sentido, gestionar mediante los distintos estamentos y dispositivos del establecimiento las necesidades que surgen a partir de las transiciones de nivel, y aquellas propias de la etapa del ciclo evolutivo, escuchando activamente sus demandas y proporcionando espacios para su óptimo desenvolvimiento.

Por último, el tercer componente con el objeto de promover el desarrollo personal y social del alumnado en concordancia con el proyecto educativo institucional, democratizando los espacios institucionales en favor de un diálogo que fomente la cohesión y fortalezca el tejido social al interior del establecimiento.

Estas orientaciones encuentran su asidero en la medida que los sujetos pueden influir sobre sus propios campos de acción, facilitando la integración de experiencias vitales, herramientas técnicas y saberes compartidos en una realidad contextual. En otras palabras, las prácticas a emplear se traducen en la capacidad de articular a los actores con su realidad material y simbólica, focalizando los esfuerzos sobre los hallazgos de factores de repitencia y potenciando los dispositivos existentes, en consideración de no sobreintervenir el espacio educativo. Dichos componentes se llevan a efecto entre la alianza familia - escuela, en la potenciación de pautas de aprendizaje y preparación para la transición y, finalmente, en la participación de la comunidad educativa y el rol docente como soporte social y emocional en la construcción personal y colectiva del estudiante.

En definitiva, la repitencia escolar no es sino la manifestación de un proceso multidimensional enmarcada en una sociedad desigual con disparidades en los aprendizajes, cuyos 
resultados académicos son su expresión individual. Su abordaje, por lo tanto, no debe reducirse tan solo a la focalización de aquellos estudiantes que reprueban y no cumplen con los mínimos normativos, sino que debe responsabilizar a la comunidad en su conjunto, relevando el valor de la democracia y la colaboración en la búsqueda de beneficios comunes.

\section{Agradecimientos / Acknowledgments:}

Expreso mi agradecimiento a los docentes de la Maestría en Psicología Social de la Unidad de posgrado de la Universidad Valparaíso, Chile.

\section{Conflicto de intereses / Competing interests:}

El autor declara que no incurre en conflictos de intereses.

Rol de los autores / Authors Roles:

No aplica.

\section{Fuentes de financiamiento / Funding:}

El autor declara que no recibió un fondo específico para esta investigación.

Aspectos éticos / legales; Ethics / legals:

El autor declara no haber incurrido en aspectos antiéticos, ni haber omitido aspectos legales en la realización de la investigación.

\section{REFERENCIAS BIBLIOGRÁFICAS}

Ander-Egg, E. (2008). Métodos y técnicas de investigación social II. La ciencia: su método y la expresión del conocimiento científico. Buenos Aires: Lumen.

Andréu, J. (2000). Las técnicas del análisis de contenido: una revisión actualziada. Granada: Universidad de Granada.

Ardila, A., Gusmá, E., Matute, E., San Martín, A., \& Rosselli, M. (2009). Influencia del nivel educativo de los padres, el tipo de escuela y el sexo en el desarrollo de la atención y la memoria. Revista Latinoamericana de Psicología, 41(2), 257-276. Obtenido de https://www.redalyc.org/pdf/805/80511496006.pdf

Bellei, C. (2007). Expansión de la educación privada y mejoramiento de la educación en Chile. Evaluación a partir de la evidencia. Pensamiento Educativo, 40(1), 1-21. Obtenido de http://pensamientoeducativo.uc.cl/index.php/pel/article/view/408/837

Bellei, C., Poblete, X., Sepúlveda, P., Orellana, V., \& Abarca, G. (2013). Situación educativa de América Latina y el Caribe: hacia la educación de calidad para todos al 2015. Informe UNESCO. Santiago: Imbuche Ltda.

Bellei, C., Valenzuela , J., Vanni, X., \& Contreras, D. (2014). Lo aprendí en la escuela. ¿Cómo se logran procesos de mejoramientoe escolar? Santiago: Universidad de Chile.

Berroeta, H. (2011). Apuntes para una intervención psicosocial con incidencia. Castalia(19), 3750. Obtenido de http://bibliotecadigital.academia.cl/bitstream/handle/123456789/2868/3750.pdf? sequence $=1 \&$ is Allowed $=y$

Centro UC Estudios Políticas y Prácticas de Educación. (2016). Revisión de literatura sobre políticas y normativa de promoción y retención, y su impacto en el aprendizje. Consultoría para MINEDUC-UNESCO. Santiago, Chile: Autor. 
Chile, Ministerio de Educación . (2018). Agencia de la Calidad de la Educación. Obtenido de Categoria de desempeño 2018: resultados y análisis. Obtenido de http://archivos.agenciaeducacion.cl/Policy_brief_CD_2018.pdf

Chile, Ministerio de Educación. (2019). Indicadores de la educación 2018. Obtenido de https://centroestudios.mineduc.cl/publicaciones-ce/publicaciones-estadisticas2/publicaciones-nacionales/

Chile, Ministerio de Educación. (2019b). Informe del sistema educacional con análisis de género 2017. Santiago: Autor, Centro de Estudios.

Creswell, J., \& Plano, V. (2010). Designing and conducting mixed methods research. Calif: SAGE Publications.

Dari, N., Cervini, R., \& Quiroz, S. (2019). Repitencia escolar y desempeño en ciencias en Argentina. Estudio multinivel con base en datos de PISA 2015. Revista de Educación, $O(16), \quad 55-79 . \quad$ Obtenido de https://fh.mdp.edu.ar/revistas/index.php/r_educ/article/view/3074/3333

Dávila, O., Ghiardo, F., \& Medrano, C. (2008). Los desheredados. Trayectorias de vida y nuevas condiciones juveniles $\left(4^{\mathrm{a}} \mathrm{ed}\right.$.). Valparaíso: CIDPA.

Decreto $\mathrm{N}^{\circ}$ 83. Diario Oficial de la República de Chile, Santiago, Chile, 20 de febrero de 2018.

Decreto $\mathrm{N}^{\circ}$ 83. Diario Oficial de la República de Chile, Santiago, Chile, 6 de marzo de 2001.

Decreto $N^{\circ}$ 112. Diario Oficial de la República de Chile, Santiago, Chile, 20 de abril de 1999.

Decreto $\mathrm{N}^{\circ}$ 511. Diario Oficial de la República de Chile, Santiago, Chile, 8 de mayo de 1997.

Díaz, L., Torruco, U., Martínez, M., \& Varela, M. (2013). La entrevista, recurso fexible y dinámico. Investigación en educación médica, 7(2), 162-167. Obtenido de http://www.scielo.org.mx/pdf/iem/v2n7/v2n7a9.pdf

Donoso, S., \& Hawes, G. (2002). Eficiencia escolar y diferencias socioeconómicas: a propóstio de los resultados de las pruebas de medición de la calidad de la educación en Chile. Educação e Pesquisa, São Paulo, 28(2), 25-39. doi:10.1590/S1517-97022002000200003

Espinoza, O., Castillo, D., González, L., \& Loyola, J. (2012). Factores familiares asociados a la deserción escolar en Chile. Revista de Ciencias Sociales (RCS) , 13(1), 136-150. doi:10.4067/S0718-07052014000100006

França-Tarragó, O. (2005). Ética para psicólogos: introducción a la psicoética. Montevideo: Descleé de Brower.

García, M. V., Alvarado, M. J., \& Jiménez, A. (2000). La predicción del rendimiento académico: regresión lineal versus regresión logística. Psicothema, 12(2), 248-252. Obtenido de http://www.psicothema.com/pdf/558.pdf 
Ikeda, M., \& García, E. (2014). Grade repetition: A comparative study of academic and nonacademic consequences. OECD Journal: Economic Studies, 2013(1), 269-315. doi:10.1787/19952856

Iñiguez, L. (1999). Investigación y evaluación cualitativa: bases teóricas y conceptuales. Revista Atención Primaria, 23(8), 496-502. Obtenido de http://www.unidaddocentemfyclaspalmas.org.es/resources/1+Aten+Primaria+1999.+IC+ Bases+Teoricas+y+Conceptos.pdf

Laboratorio Latinoamericano de la Evaluación de la Calidad de la Educación. (2015). Informe de Resultados TERCE: factores asociados. Santiago: UNESCO. Obtenido de http://archivos.agenciaeducacion.cl/8_Informe_internacional_de_resultados_Factores_as ociados_TERCE_2013.pdf

Martínez, V. (2016). Efecto de la repitencia sobre años de escolaridad alcanzados (Tesis de Magíster). Universidad de Chile. Obtenido de http://repositorio.uchile.cl/bitstream/handle/2250/144081/Mart\%C3\%ADnez\%20Garrido $\% 20$ Vania.pdf?sequence $=1 \&$ isAllowed $=y$

Meneses, J., \& Rodríguez, D. (2011). El cuestionario y la entrevista. Barcelona : Editorial UOC.

Montenegro, M. (2001). Conocimiento, agentes y articulaciones: una mirada situada a la intervención social. Obtenido de http://hdl.handle.net/10803/5410

Montenegro, M. (2002). El Cambio Social Posible: Reflexiones en torno a la intervención social. En ARCIS. Políticas Sujetos y Resistencias. Debates y Críticas de Psicología Social. Cuadernos de Psicología Social N${ }^{\circ}$. (pp.229-236). Universidad Arcis. Santiago.

Oliva, M. (2010). Política educativa chilena 1965-2009. ¿Qué oculta esa trama? Revista Brasileira de Educação , 15(44), 311-328. doi:10.1590/S1413-24782010000200008

Organización para la Cooperación y el Desarrollo Económico. (2016). Pisa 2015. Resultados claves. Autor: OCDE.

Papalia, D., Feldman, R., Martorell, G., Berber , E., \& Vásquez, M. (2012). Desarrollo Humano (12 ed.). México D.F.: McGraw-Hill Interamericana.

Passolini, M. (2011). La repitencia como problema de (in)justicia social: un análisis de estrategias alternativas en Brasil y Argentina. VI Jornadas Jóvenes de Investigadores. Instituto de investigaciones Gino Germani, Facultad de Ciencias Sociales, Universidad de Buenos Aires. Buenos Aires. Obtenido de https://www.aacademica.org/000-093/392.pdf

Puga, I. (2011). Escuela y estratificación social en Chile: ¿Cuál es el rol de la municipalización y la educación particular subvencionada en la reproducción de la desigualdad social? Estudios Pedagógicos, 37(2), 213-232. doi: 10.4067/S0718-07052011000200013

Raczynski, D., Hernández, M., Kegevic, L., \& Rocco, R. (2011). El paso de la enseñaza básica a la media en estratos bajos: Un reto a la igualdad de oportunidades educativas. Santiago: Fondo de Investigación y Desarrollo de la Educación - FONIDE, Ministerio de Educación. 
Obtenido $\quad$ de $\quad$ https://centroestudios.mineduc.cl/wpcontent/uploads/sites/100/2017/07/Informe-Final-Dagmar-Raczynski-F511083.pdf

Rodriguez, M., \& Mora, R. (2001). Análisis de regresión múltiple. Técnicas de Investigación Social II. Alicante: Universidad de Alicante.

Román, M. (2013). Factores asociados al abandono y la deserción escolar en América Latina: una mirada en conjunto. Revista Iberoamericana sobre calidad, eficiencia y cambio en educación, 11(2), 33-59. Obtenido de https://www.redalyc.org/pdf/551/55127024002.pdf

Rossano, A. (2006). El pasaje de la primaria a la secundaria como transición educativa. En F. Terigi, Diez miradas sobre la escuela primaria (págs. 295-317). Buenos Aires: Siglo XXI/Fundación OSDE.

Sánchez, A., Reyes, F., \& Villarroel, V. (2016). Participación y expectativas de los padres sobre la educación de sus hijos en una escuela pública. Estudios Pedagógicos , 42(3), 347-367. doi: 10.4067/S0718-07052016000400019

Sandoval, J. (2013). Una perspectiva situada de la investigación cualitativa en ciencias sociales. Cinta Moebio(46), 37-46. doi: 10.4067/S0717-554X2013000100004

Schwerdt, G., West, M., \& Winters, M. (2015). The effects of test-based retention on studentoutcomes over time: Regression discontinuity evidence from Florida. Journal of Public Economics, 154-169. doi: 10.1016/j.jpubeco.2017.06.004

Superintendencia de Educación Escolar. (2016). Resguardo de derechos en la escuela. Orientaciones para la aplicación de la normativa educacional. https://supereduc.cl/images/Resguardo_de_Derechos_en_la_EscuelaOrientaciones_para_la_Aplicaci\%C3\%B3n_de_la_Normativa_Educacional.pdf

Torres, J., Acevedo, D., \& Gallo, L. (2015). Causas y consecuencias de la deserción y repitencia escolar: una revisión general en el contexto latinoamericano. Cultura Educación y Sociedad 6(2), 157-187. Obtenido de https://core.ac.uk/download/pdf/230171172.pdf

Treviño, E., \& Donoso, F. (2010). Agrupación de escuelas para intervenciones de política: análisis del caso chileno. Santiago: Universidad Diego Portales.

Valdés, A., \& Urías, M. (2011). Creencias de padres y madres acerca de la participación en la educación de sus hijos. Perfiles Educativos, 33(134), 99-114. Obtenido de http://www.scielo.org.mx/pdf/peredu/v33n134/v33n134a7.pdf

Villalobos, C., \& Béjares, C. (2017). Políticas de promoción y repitencia en Chile: recomendaciones desde una mirada comparada. CEPPE Policy Brief $\mathrm{N}^{\circ} 14$, CEPPE UC. Obtenido de http://ceppe.uc.cl/images/contenido/policy-briefs/CEPPE_N14Politicas_de_Promocion_y_Repitencia_en_Chile-

Recomendaciones_desde_una_Mirada_Comparada.pdf 